\title{
CircPVTI Regulates Cell Proliferation, Apoptosis and Glycolysis in Hepatocellular Carcinoma via miR-377/TRIM23 Axis
}

This article was published in the following Dove Press journal: Cancer Management and Research

\author{
$\mathrm{Nan} \mathrm{Bu}^{\mathrm{l}, *}$ \\ Zheng Dong ${ }^{2} *$ \\ Lingfeng Zhang ${ }^{3}$ \\ Weibo $\mathrm{Zhu}^{4}$ \\ Furong $\mathrm{Wei}^{5}$ \\ Sheng Zheng ${ }^{6}$ \\ 'Department of Gastroenterology, \\ Chinese Medicine Hospital of Jiamusi \\ City, Heilongjiang Province, Jiamusi, \\ Heilongjiang, People's Republic of China; \\ 2Department of General Surgery, Ningbo \\ Mingzhou Hospital, Ningbo, Zhejinag, \\ People's Republic of China; ${ }^{3}$ Department \\ of Hepatobiliary Surgery, The First \\ People's Hospital of Huaihua City, \\ Huaihua, Hunan, People's Republic of \\ China; ${ }^{4}$ Department of Medical \\ Technology, Qujing Medical College, \\ Qujing, Yunan, People's Republic of \\ China; ${ }^{5}$ Department of Gastroenterology, \\ Central Hospital of Haining, Haining, \\ Zhejinag, People's Republic of China; \\ ${ }^{6}$ Department of Gastroenterology, The \\ Third People's Hospital of Yunnan \\ Province, Kunming, Yunnan, People's \\ Republic of China
}

*These authors contributed equally to this work
Background: Recent studies reported that circular RNAs (circRNAs) exert essential functions in hepatocellular carcinoma (HCC) progression. However, the expression profile and function of circular RNA PVT1 (circPVT1) in HCC are not fully addressed. Thus, we aimed to probe into the function of circPVT1 in HCC development.

Methods: The levels of circPVT1, microRNA-377 (miR-377) and transcripts encoding tripartite motif containing 23 (TRIM23) were determined by qRT-PCR. The stability and localization of circPVT1 were examined by RNase R digestion assay and subcellular fraction assay, respectively. Cell proliferation and apoptosis were evaluated by MTT assay and flow cytometry analysis, respectively. The relationship between miR-377 and circPVT1 or TRIM23 was determined by dual-luciferase reporter assay and RNA immunoprecipitation (RIP). The protein expression of TRIM23 was measured by Western blot. The glycolysis level was assessed by specific kits and Seahorse Extracellular Flux Analyzer XF96. The function of circPVT1 in vivo was investigated in a murine xenograft model.

Results: CircPVT1 and TRIM23 levels were elevated, while miR-377 was decreased in HCC. CircPVT1 knockdown restrained proliferation and glycolysis, but enhanced apoptosis in HCC cells. CircPVT1 could bind to miR-377 and inhibition of miR-377 restored circPVT1 knockdown-mediated effect on HCC cells. TRIM23 was certified as a target of miR-377, and TRIM23 upregulation overturned the influence of miR-377 upregulation or circPVT1 silence on HCC progression. Moreover, circPVT1 knockdown restrained tumor growth in HCC in vivo.

Conclusion: CircPVT1 aggravated the progression of HCC by upregulating TRIM23 via sponging miR-377.

Keywords: hepatocellular carcinoma, circPVT1, miR-377, TRIM23

\section{Introduction}

Hepatocellular carcinoma (HCC) is a prevalent and lethal cancer worldwide, which ranks as the sixth of cancer-related death with a fairly high incidence and mortality. ${ }^{1}$ Although the diagnosis and treatment of HCC have been improved in recent years, the overall survival rate is still low because of the high risk for disease metastasis and recurrence. Hence, it is significant to uncover the underlying molecular mechanism of HCC progression.

Circular RNAs (circRNAs) with closed single-stranded continuous rings have been demonstrated to take part in the regulation of multiple malignant tumors, including HCC. ${ }^{2,3}$ For example, circPTPRM could promote HCC cell proliferation, migration and invasion. ${ }^{4}$ CircVAPA was abnormally upregulated in HCC and could
Department of Gastroenterology, The Third People's Hospital of Yunnan Province, 292 Beijing Road, Guandu District, Kunming City, Yunnan Province, People's Republic of China Tel +86-87I-63I96247

Email x5z25a@163.com 
accelerate proliferation of HCC cells. ${ }^{5}$ Circular RNA PVT1 (circPVT1) has been verified to play a promoting role in various cancers, including oral squamous cell carcinoma, ${ }^{6}$ esophageal carcinoma ${ }^{7}$ and colorectal cancer. ${ }^{8}$ Likewise, circPVT1 has also been reported to be upregulated in $\mathrm{HCC}$ and expedite proliferation and migration in HCC cells. ${ }^{9}$ However, the related reports about the expression and potential role of circPVT1 in HCC are inadequate.

MicroRNAs (miRNAs) are small noncoding RNAs and can affect mRNA expression via binding to its $3^{\prime}$ untranslated region (3'UTR). ${ }^{10}$ Numerous studies have shown that miR-377 could affect cancer progression in different types of cancers through acting as cancerpromoting gene ${ }^{11,12}$ or tumor suppressor. ${ }^{5,13}$ For instance, overexpression of miR-377 could suppress HCC cell progression. ${ }^{14,15}$ Transcripts encoding tripartite motif containing 23 (TRIM23) is an ubiquitin ligase belonging to the tripartite motif (TRIM) family and has been certified to participate in tumor development. ${ }^{16}$ However, the relationship between miR-377 and TRIM23 and the specific mechanism underlying HCC progression has not been documented.

In the present study, the detailed function of circPVT1 in vitro and in vivo was investigated in HCC. Besides, a circRNA-miRNA-mRNA functional network was constructed to clarify the potential mechanism of HCC progression.

\section{Patients and Methods}

\section{Patients and Tissue Samples}

Twenty-six pairs of HCC tissues and the adjacent normal tissues were collected from HCC patients at the Central Hospital of Jiamusi City and the tissue samples were stored at $-80^{\circ} \mathrm{C}$ until use. The patients had not accepted with any preoperative anti-tumor treatments. This study had obtained approval from the Ethics Committee of the Central Hospital of Jiamusi City. All participants signed the written informed consent.

\section{Cell Culture}

The normal human liver cell line (THLE-2) and HCC cell line (SNU-387) were purchased from the American Type Culture Collection (ATCC, Manassas, VA, USA). HCC cell line (Huh7) was bought from Procell (Wuhan, China). Huh7 cells were maintained in Dulbecco's modified Eagle's medium (DMEM, Gibco, Carlsbad, CA,
USA), and SNU-387 and THLE-2 cells were incubated in Roswell Park Memorial Institute 1640 (RPMI-1640) medium (Gibco) and Bronchial Epithelial Basal medium (BEBM, HY StemCell, Chongqing, China), respectively. All the media supplemented with $1 \%$ penicillinstreptomycin (Gibco) and 10\% fetal bovine serum (FBS; Gibco). All cells were cultured in a humidified incubator at $37^{\circ} \mathrm{C}$ with $5 \% \mathrm{CO}_{2}$.

\section{Cell Transfection}

Small interfering RNA (siRNA) against circPVT1 (sicircPVT1) and its corresponding negative control (si-NC), miR-377 mimic and the control (miRNA NC), miR-377 inhibitor and the control (inhibitor NC), TRIM23 overexpression vector (pcDNA-TRIM23) and the control (pcDNA-Control), and lentivirus-mediated short hairpin RNA against circPVT1 (sh-circPVT1) and its control (shNC) were obtained from Genepharma (Shanghai, China). The above plasmids or oligonucleotides were transfected into HCC cells by Lipofectamine 2000 Reagent (Invitrogen, Carlsbad, CA, USA).

\section{Quantitative Real-Time Polymerase Chain Reaction (qRT-PCR)}

Trizol reagent (Invitrogen) was utilized to extract total RNA. Briefly, the total RNA was reverse-transcribed into complementary DNA using PrimeScript ${ }^{\mathrm{TM}}$ RT reagent Kit (Takara, Dalian, China) or miRNA 1st Strand cDNA Synthesis Kit (Vazyme, Nanjing, China). Then, the amplified transcript expression of the relative specific genes was determined utilizing BeyoFast ${ }^{\mathrm{TM}}$ SYBR Green qPCR Mix (Beyotime, Shanghai, China). The relative expression was determined with the $2^{-\Delta \Delta \mathrm{Ct}}$ method. The expression of circPVT1, linear RNA PVT1 and TRIM23 was normalized by glyceraldehyde 3-phosphate dehydrogenase (GAPDH), while miR-377 expression was normalized to the level of U6. The primer sequences of the relative genes were listed as follows $\left(5^{\prime}-3^{\prime}\right)$ : circPVT1, GGTTCCAC CAGCGTTATTC (Forward, F) and CAACTTCCT TTGGGTCTCC (Reverse, R); linear RNA PVT1, TTCAGCACTCTGGACGGACTT (F) and TATGGCATG GGCAGGGTAG' (R); miR-377, TGGTGTCGTGGAG TCG (F) and ACACTCCAGCTGGGATCACACAA AGGCA (R); TRIM23, TTGGAATGGCTCACACA GAAC (F) and ACATGGGCATCAACAACAC (R); GAPDH, GAAGGTGAAGGTCGGAGTC (F) and GAAGATGGTGATGGGATTTC (R); U6, CTCGCTTCG 
GCAGCACA (F) and AACGCTTCACGAATTTGCGT (R); $\beta$-actin: TGGACTTCGAGCAGGAAATGG (F) and ACGTCGCACTTCATGATCGAG (R); U2, AATACCAG GTCGATGCGTG (F) and CCTTTTGGCTAAGATCAAG TGTAG (R).

\section{RNase R Digestion}

Total RNA isolated from SNU-387 and Huh7 cells was treated with RNase R (Solarbio, Beijing, China) or not (MOCK), with MOCK serving as control. After incubation for $15 \mathrm{~min}$ at $37^{\circ} \mathrm{C}$, the samples were used for qRT-PCR assay.

\section{Subcellular Fraction Assay}

Cytoplasmic and Nuclear RNA Purification Kit (Norgen Biotek, Thorold, Canada) was applied to separate the nuclear and cytoplasmic RNA of SNU-387 and Huh7 cells following the protocols to measure the localization of linear and circular RNA. The expression patterns of circPVT1, linear RNA, $\beta$-actin and U2 in cytoplasmic and nucleus fractions were determined by qRT-PCR. U2 and $\beta$-actin were served as controls of nuclear fraction and cytoplasm fraction, respectively.

\section{3-(4, 5-Dimethyl-2-Thiazolyl)-2, 5-Diphenyl-2-H-Tetrazolium Bromide (MTT) Assay}

Cell proliferation was determined by MTT assay. Briefly, SNU-387 and Huh7 cells were added with $20 \mu \mathrm{L}$ MTT ( $5 \mathrm{mg} / \mathrm{mL}$; Sangon, Shanghai, China) at $0 \mathrm{~h}, 24 \mathrm{~h}, 48 \mathrm{~h}$ and $72 \mathrm{~h}$ after transfection and incubation for another 4 h. Then, $150 \mu \mathrm{L}$ dimethyl sulfoxide (DMSO; Solarbio) was added to dissolve the formazan crystals. The optical density (OD) value at $490 \mathrm{~nm}$ was examined with a microplate reader (BioTek Instruments, Winooski, VT, USA).

\section{Flow Cytometry Analysis}

Annexin V-fluorescein isothiocyanate (FITC)/propidium iodide (PI) Apoptosis Detection Kit (BD Pharmingen, San Diego, CA, USA) was used to test apoptosis of SNU-387 and Huh7 cells. In brief, cells were collected at $48 \mathrm{~h}$ after transfection. Then, $5 \mu \mathrm{L}$ AnnexinV-FITC and 5 $\mu \mathrm{L}$ PI were used to incubate cells in the darkness for 15 min. Apoptotic cells were tested with a FACScan flow cytometer (Becton Dickinson, Mountain View, CA, USA).

\section{Dual-Luciferase Reporter Assay}

The wide-type sequences of circPVT1 or TRIM23 3' UTR containing predicted binding sequences of miR-377 and mutant sequences of circPVT1 or TRIM23 3' UTR were constructed and cloned into the pGL3 promoter vectors (Invitrogen) to generate WT-circPVT1, MUT-circPVT1, WTTRIM23, MUT-TRIM23 luciferase reporter vectors, respectively. Then, the above reporter vectors and miR-377 mimic or miRNA NC were co-transfected into SNU-387 and Huh7 cells. Finally, Dual-Luciferase Reporter Assay Kit (Promega, Madison, WI, USA) was used to detect the relative luciferase activity.

\section{RNA Immunoprecipitation (RIP) Assay}

RIP assay was conducted using the Magna RIP $^{\mathrm{TM}}$ RNA Binding Protein Immunoprecipitation Kit (Millipore, Bedford, MA, USA). In brief, SNU-387 and Huh7 cells were collected and lysed in RIP buffer, and then incubated with magnetic beads conjugated with antibody against Argonaute2 (Ago2) (ab32381) (Anti-Ago2; Abcam, Cambridge, MA, USA) or immunoglobulin G (IgG) antibody (ab133470) (Anti-IgG; Abcam). The RNA enrichment of circPVT1 and miR-377 in the magnetic beads was detected by qRT-PCR.

\section{Western Blot Assay}

The involved total protein was isolated using RIPA lysis buffer (Beyotime). Then, 10\% sodium dodecyl sulfonatepolyacrylamide gel (SDS-PAGE; Solarbio) electrophoresis was adopted to separate total protein. Next, the protein was transferred onto polyvinylidene difluoride membranes (PVDF; Bio-Rad, Hercules, CA, USA) and blocked in skim milk for $2 \mathrm{~h}$. Afterwards, the primary antibody against TRIM23 (orb555874) (1:1000; Biorbyt, Cambridge, UK) or GAPDH (1:1000; Biorbyt) (orb38655) was used to incubate the membranes at $4^{\circ} \mathrm{C}$ overnight. Subsequently, the membrane was incubated with secondary antibody (ab6721; 1:5000; Abcam) at room temperature for $1 \mathrm{~h}$. Finally, the signals of protein were visualized using ECL Chemiluminescence kit (Pierce, Rockford, IL, USA) and analyzed by ImageJ (NIH, Bethesda, MD, USA).

\section{Determination of Glucose Consumption and Lactate Production}

Glucose Assay Kit (Abcam) and Lactate Assay Kit (SigmaAldrich, St. Louis., MO, USA) were used to determine glucose consumption and lactate production in SNU-387 
and Huh7 cells, respectively. The experiments were performed based on the instructions.

\section{Extracellular Acidification (ECAR) and Oxygen Consumption Rate Assays (OCR)}

The Seahorse Extracellular Flux Analyzer XF96 (Seahorse Bioscience, North Billerica, MA, USA) was used to calculate ECAR and OCR in HCC cells. After transfection, cells $\left(2 \times 10^{4}\right.$ per well $)$ were seeded into the XF96-well plate and cultured overnight. For ECAR determination, Glucose, Oligomycin (oxidative phosphorylation inhibitor), and 2-deoxyglucose (2-DG; glycolytic inhibitor) were sequentially injected into HCC cells. For OCR determination, cells were sequentially injected with Oligomycin, FCCP (reversible inhibitor of oxidative phosphorylation), and Rotenone/ antimycin A (Rote/AA). Finally, the data were analyzed, and ECAR detection and OCR detection were noted as $\mathrm{mPH} /$ $\mathrm{min}$ and $\mathrm{pmol} / \mathrm{min}$, respectively.

\section{Murine Xenograft Model}

To establish HCC murine xenograft model, the 4-6 weeks old BALB/c nude mice purchased from Beijing Vital River Laboratory Animal Technology (Beijing, China) were used. SNU-387 cells stably transfected with sh-circPVT1 or sh-NC were subcutaneously injected into the right flanks of the nude mice. After injection for 7 days, the length (L) and width (W) of tumors were measured every 4 days using a caliper and tumor volume was assessed using the formula: $\left(\mathrm{L} \times \mathrm{W}^{2}\right)$ / 2. The mice were sacrificed at 27 days after injection and the tumor weight was calculated. The experimentation on animals was approved by the Ethics Committee of Animal Research of the Central Hospital of Jiamusi City. The experiments were carried out in accordance

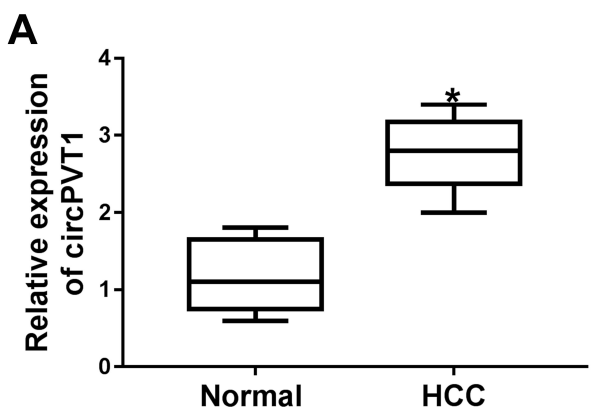

with the National Guidelines for Experimental Animal Welfare (the Ministry of Science and Technology, China).

\section{Statistical Analysis}

All data in this study obtained from at least three independent experiments were displayed as the mean \pm the standard deviations (SD) and analyzed by SPSS 18.0 software. Difference analysis between two groups or among multiple groups was conducted using Student's $t$ test or one-way analysis of variance (ANOVA). $P$-value less than 0.05 was defined as a statistically significant difference.

\section{Results}

\section{CircPVTI Was Upregulated in HCC Tissues and Cells}

Firstly, circPVT1 level in 26 paired tumorous tissues and the adjacent normal tissues from HCC patients was detected by qRT-PCR. The result suggested that circPVT1 was significantly upregulated in HCC tissues relative to that in normal tissues (Figure 1A). Besides, we found that the level of circPVT1 was elevated in HCC cells (SNU-387 and Huh7) in comparison to THLE-2 cells (Figure 1B). Overall, circPVT1 might be associated with the development of HCC.

\section{Silencing of circPVTI Suppressed Cell Proliferation and Accelerated Apoptosis of HCC Cells}

Next, we examined the stability of circPVT1 by RNase $\mathrm{R}$ digestion assay and the data displayed that RNase $\mathrm{R}$ treatment distinctly suppressed linear PVT1 mRNA level in SNU-387 and Huh7 cells, but the level of circular RNA PVT1 (circPVT1) was not affected, indicating that

B

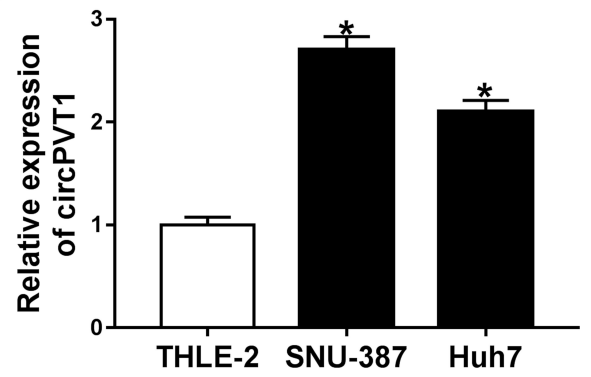

Figure I CircPVTI was upregulated in HCC tissues and cells. (A) The expression of circPVTI in 26 pairs of HCC tissues and the adjacent non-tumor tissues was detected by qRT-PCR. (B) The expression of circPVTI in HCC cells (SNU-387 and Huh7) and normal liver cell lines (THLE-2) was examined by qRT-PCR. *P<0.05. 
circPVT1 was indeed circRNA (Figure 2A). Subsequently, subcellular fraction assay manifested that circPVT1 was mainly located in the cytoplasm, and its distribution proportion in the cytoplasm was larger than that of linear RNA (Figure 2B and $\mathrm{C}$ ). To explore the function of circPVT1 in HCC, si-circPVT1 or si-NC was transfected into SNU-387 and Huh7 cells to knock down circPVT1 expression. As expected, circPVT1 expression was distinctly declined after transfection with si-circPVT1, while linear PVT1 mRNA level was not changed (Figure 2D and E). All these exhibited the successful knockdown efficiency of circPVT1. Meanwhile, MTT assay demonstrated that circPVT1 deletion repressed the proliferation of SNU-387 and Huh7 cells (Figure 2F and G). Moreover, the apoptosis was dramatically enhanced in SNU-387 and Huh7 cells by si-circPVT1 transfection, as illustrated by flow cytometry (Figure 2H). All these data demonstrated that circPVT1 served as a cancer-promoting gene in HCC progression.

\section{Knockdown of circPVTI Suppressed Proliferation and Enhanced Apoptosis via Binding to miR-377 in HCC Cells}

To study the underlying mechanism of circPVT1 in the regulation of $\mathrm{HCC}$, the direct miRNA targets of circPVT1 were predicted by online software starBase. As depicted in Figure 3A, circPVT1 contained the potential binding sequence of miR-377. Afterwards, the dual-luciferase reporter assay was performed and the result exhibited that co-transfection of WTcircPVT1 and miR-377 mimic caused a significant decline of the luciferase activity compared to WTcircPVT1 and miRNA NC group, while no obvious change was observed in MUT-circPVT1 groups (Figure 3B and C). Meanwhile, RIP assay presented that circPVT1 and miR-377 were enriched in AntiAgo2 immunoprecipitates in SNU-387 and Huh7 cells relative to control Anti-IgG group (Figure 3D and E), which further confirmed the combination between circPVT1 and miR-377. Moreover, our data showed that miR-377 was decreased in HCC tissues (Supplement Figure 1A). Furthermore, miR-377 was upregulated by circPVT1 silence in SNU-387 and Huh7 cells (Figure 3F). qRT-PCR revealed that miR377 expression was dropped after miR-377 inhibitor transfection (Figure 3G). Intriguingly, miR-377 inhibitor overturned the enhancement effect of circPVT1 knockdown on miR-377 expression (Figure 3H). More interestingly, the inhibitory impacts of circPVT1 silence on cell proliferation and the facilitating effect on apoptosis were restored by miR-377 inhibitor (Figure 3I-K). Together, these findings suggested that circPVT1 silence impeded cell proliferation and

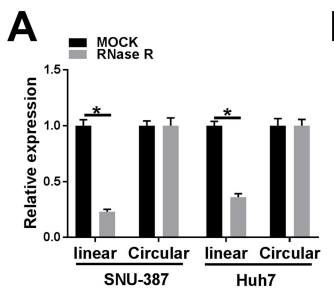

$\mathbf{F}$

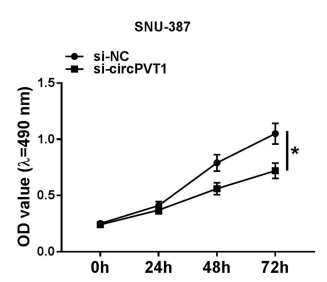

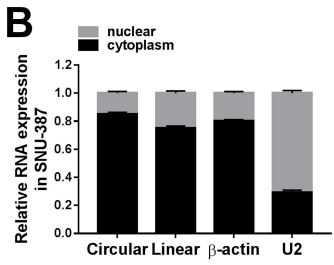

G

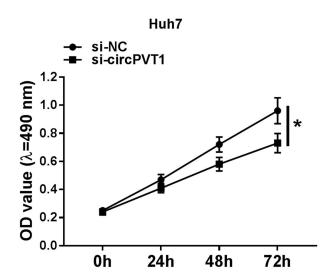

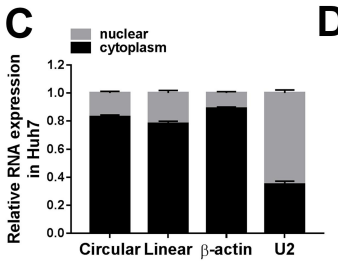

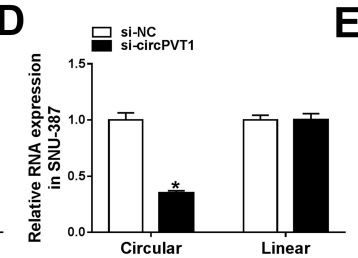

H
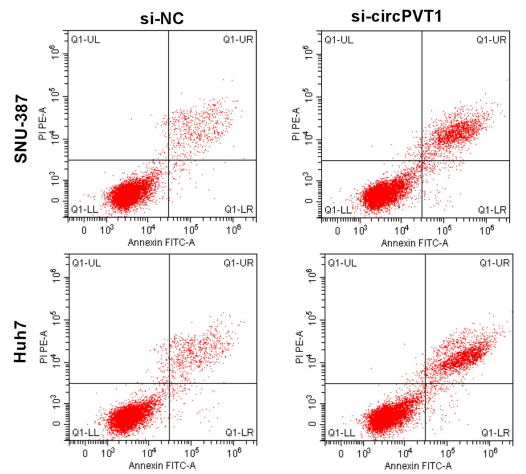
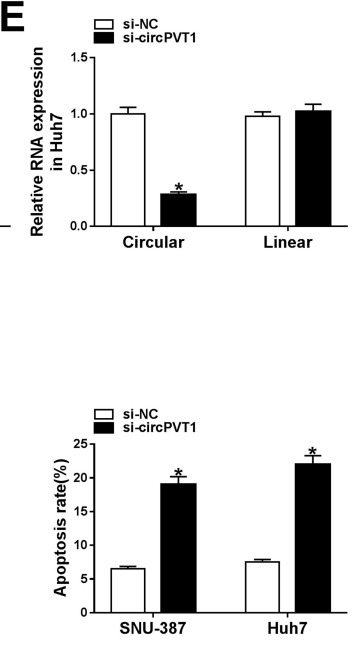

Figure 2 CircPVTI knockdown inhibited cell proliferation and induced apoptosis in HCC cells. (A) The expression of linear PVTI mRNA and circular circPVTI in SNU-387 and Huh7 cells treated or untreated with RNase R was determined by qRT-PCR. (B and C) Relative expression levels of circular RNA circPVTI, linear RNA PVTI in the nuclei and cytoplasm of SNU-387 and Huh7 cells were measured by qRT-PCR. (D-H) SNU-387 and Huh7 cells were transfected with si-circPVTI or si-NC. (D and E) The expression levels of circPVTI and linear PVTI in SNU-387 and Huh7 cells were evaluated using qRT-PCR. (F and G) The proliferation of SNU-387 and Huh7 cells was tested using MTT assay. $(\mathbf{H})$ The apoptosis rate of SNU-387 and Huh7 cells was assessed using flow cytometry. $* P<0.05$. 
A

WT-circPVT1: 5' guAAUAGCUGCAUGUGUGUGAg 3'

miR-377: $\quad$ 3' ugUUUUCAACGGAAACACACUa 5'

MUT-circPVT1: 5' guAAUAGCUGCAUGACACACUg 3'
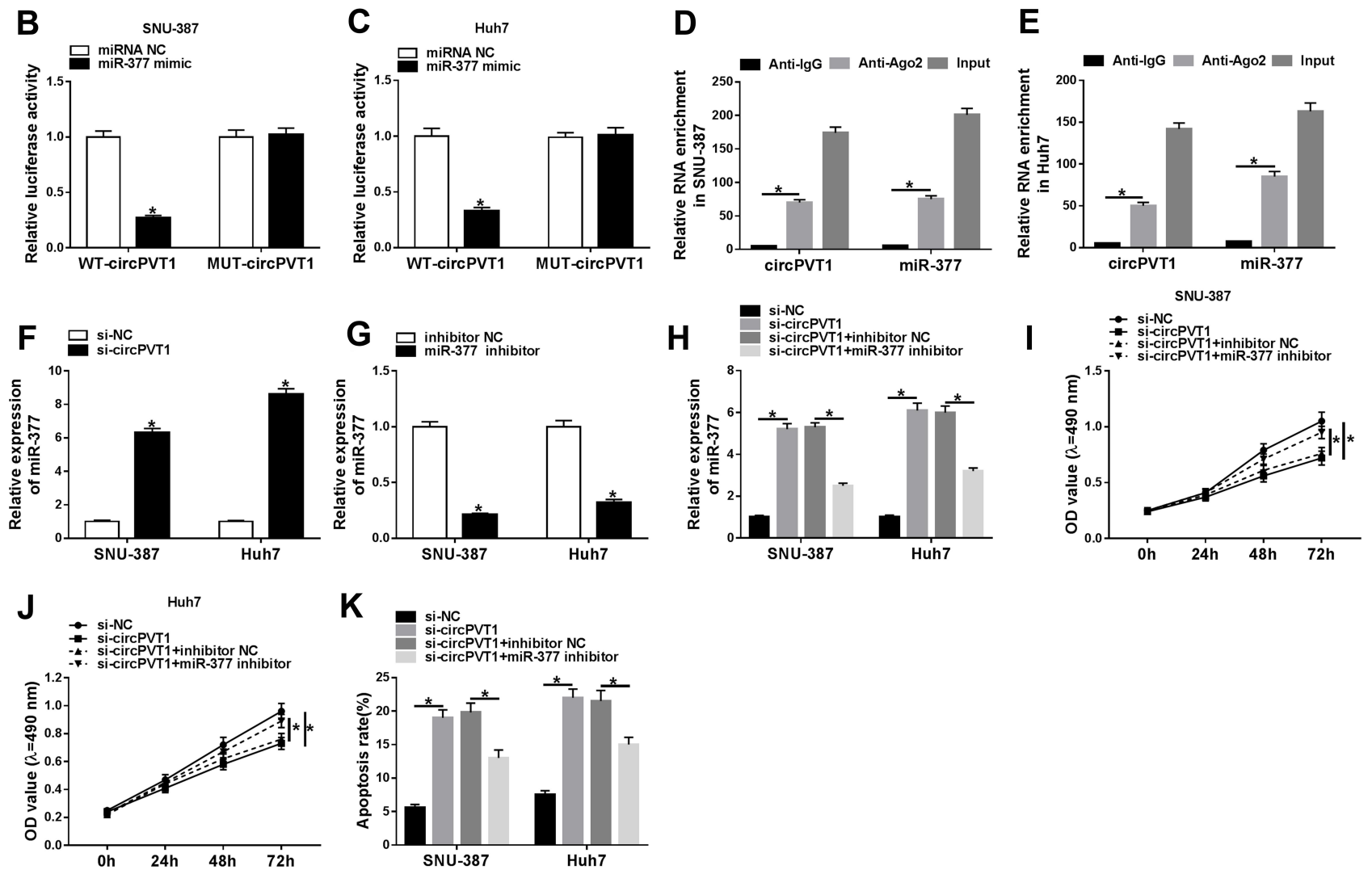

Figure 3 CircPVTI regulated proliferation and apoptosis by sponging miR-377 in HCC cells. (A) The potential binding sites between circPVTI and miR-377 were predicted by starBase. (B and C) The relative luciferase activity in SNU-387 and Huh7 cells co-transfected with WT-circPVTI or MUT-circPVTI and miR-377 mimic or miRNA NC was determined by dual-luciferase reporter assay. (D and E) RIP assay was performed to explore the enrichment of circPVTI and miR-377 in SNU-387 and Huh7 cells incubated with Anti-Ago2 or Anti-lgG antibodies. (F and $\mathbf{G})$ The expression of miR-377 in SNU-387 and Huh7 cells was examined by qRT-PCR. (H-K) SNU-387 and Huh7 cells were transfected with si-circPVTI, si-NC, si-circPVTI + miR-377 inhibitor, or si-circPVTI + inhibitor NC. (H) The expression of miR-377 in SNU-387 and Huh7 cells after transfection was tested by qRT-PCR. (I and J) Cell proliferation was detected by MTT assay. (K) The apoptosis rate of SNU-387 and Huh7 cells was assessed using flow cytometry. $* P<0.05$.

stimulated apoptosis via enhancing miR-377 expression in HCC cells.

\section{TRIM23 Overexpression Reversed} miR-377 Mimic-Mediated Effects on Cell Proliferation and Apoptosis in HCC Cells Next, the functional relationship between miR-377 and TRIM23 in HCC cells was explored. StarBase predicted that TRIM23 was a target of miR-377, and the potential binding sites between them were presented in Figure 4A. Besides, the luciferase activity was suppressed in SNU-387 and Huh7 cells after transfection with miR377 and WT-TRIM23 relative to miRNA NC and WTTRIM23 group, while the luciferase activity was insusceptible in MUT-TRIM23groups (Figure 4B and C). Our data indicated that TRIM23 was upregulated in HCC tissues (Supplement Figure 1B). Then, miR-377 mimic or miRNA NC was transfected into SNU-387 and Huh7 cells, simultaneously, and the results indicated that miR-377 was upregulated, whereas TRIM23 mRNA and protein were downregulated after overexpression of miR-377 (Figure 4D-F). Then, the transfection efficiency of TRIM23 overexpression (pcDNA-TRIM23) in SNU-387 and Huh7 cells was detected by qRT-PCR and Western blot (Figure 4G and H). MiR-377 mimic overtly restrained the proliferation and accelerated apoptosis in SNU-387 and Huh7 cells, while these effects were partly abrogated by TRIM23 overexpression 
A

WT-TRIM23-3'UTR: 5' augguAGGUGUUUGUGUGUGAu 3'

miR-377: 3 ' uguuuUCAACGGAAACACACUa 5'

MUT-TRIM23-3'UTR: 5' augguAGGUGUUUGACACACUu 3'
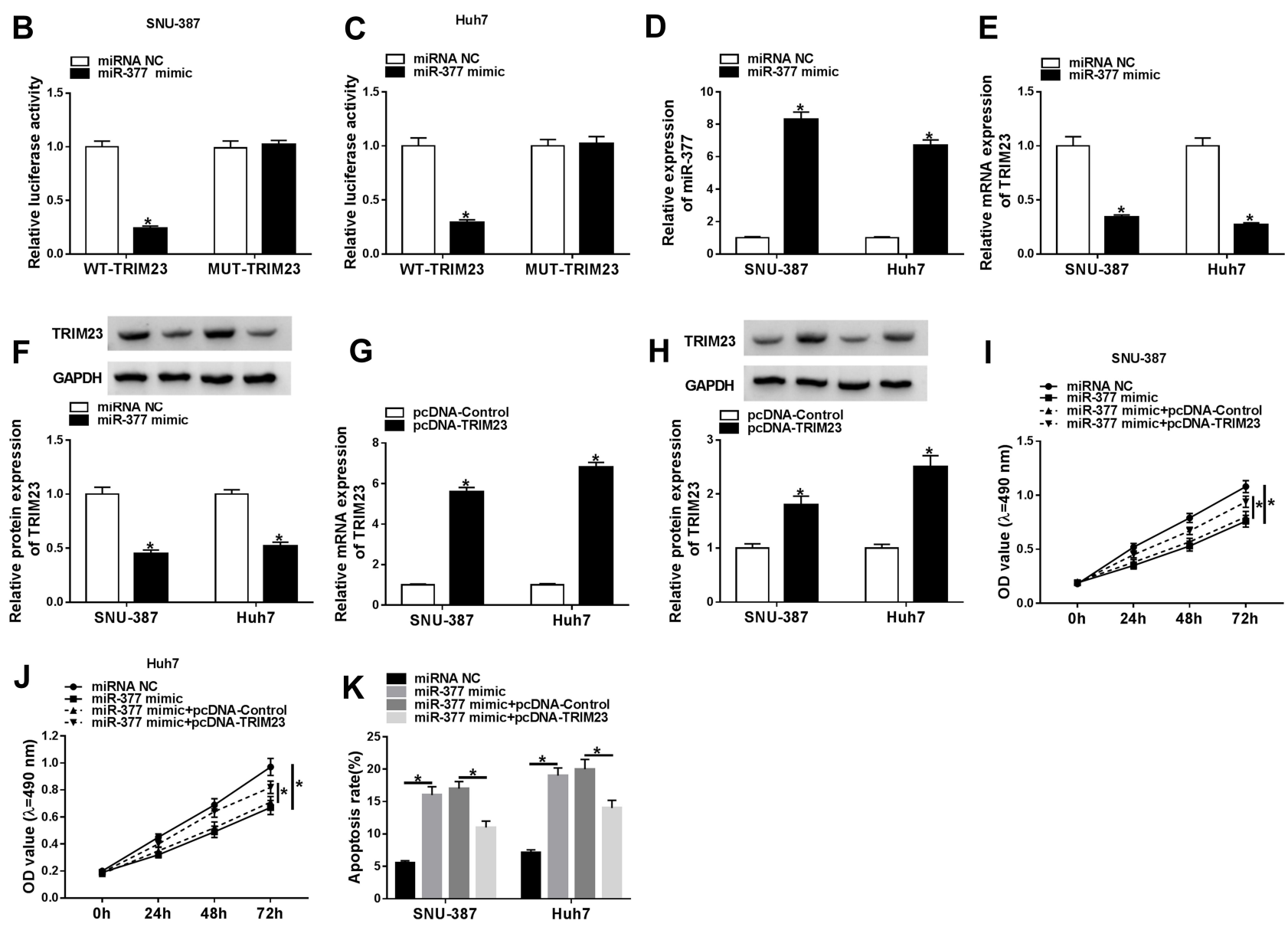

Figure 4 TRIM23 overexpression overturned the effects of miR-377 mimic on cell proliferation and apoptosis in HCC cells. (A) StarBase predicted the binding sequence between miR-377 and TRIM23. (B and C) The targeting relationship between miR-377 and TRIM23 was confirmed by dual-luciferase reporter assay. (D) The overexpression efficiency of miR-377 was checked in SNU-387 and Huh7 cells. (E-H) The mRNA and protein levels of TRIM23 in SNU-387 and Huh7 cells transfected with miR-377 mimic, pcDNA-TRIM23 or corresponding controls were measured by qRT-PCR assay or Western blot assay, respectively. (I-K) The proliferation and apoptosis of SNU-387 and Huh7 cells transfected with miR-377 mimic, miR-377 mimic + pcDNA-TRIM23 or corresponding controls were evaluated through MTT assay and flow cytometry, severally. $* P<0.05$.

(Figure 4I-K). All these findings suggested that miR377 might be involved in HCC progression through targeting TRIM23.

\section{CircPVTI Facilitated Proliferation and Suppressed Apoptosis in HCC Cells via miR-377/TRIM23 Axis}

Base on the correlation between miR-377 and circPVT1 or TRIM23, si-circPVT1, si-circPVT1 + pcDNATRIM23or matched controls was transfected into SNU387 and Huh7 cells to further testify the relationship between circPVT1 and TRIM23. As shown in Figure 5A and B, TRIM23 was dramatically depressed by circPVT1 silence both in mRNA and protein levels, while it was reacquired after TRIM23 overexpression in SNU-387 and Huh7 cells. Similarly, TRIM23 overexpression abrogated the inhibition effects on proliferation and promotion effects on apoptosis caused by sicircPVT1 (Figure 5C-E). Afterwards, we investigated the relationship among circPVT1, miR-377 and TRIM23 by examining TRIM23 expression in SNU-387 and Huh7 cells after being transfected with si-circPVT1, si-circPVT1 + miR-377 inhibitor or matched controls. 

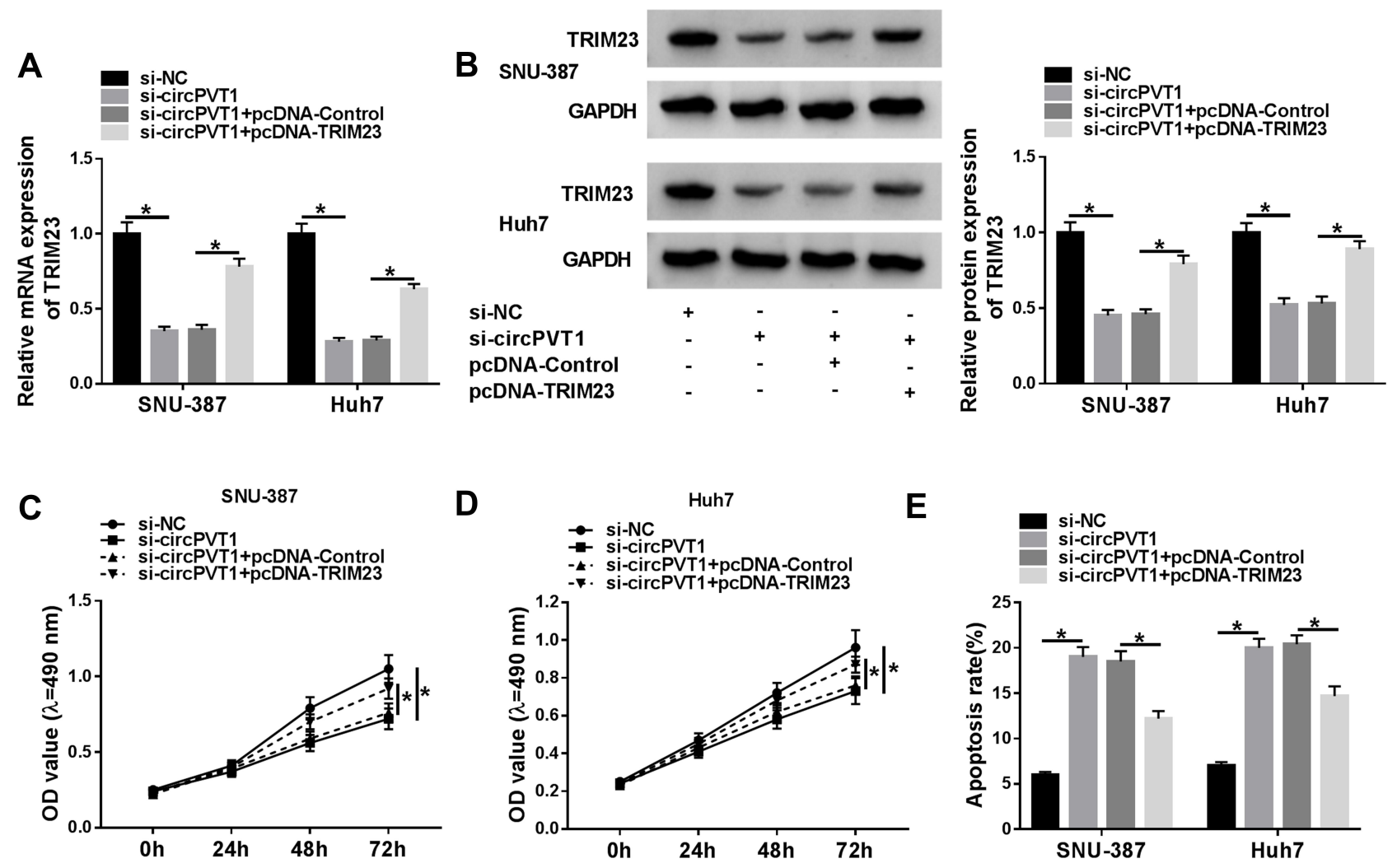

Figure 5 TRIM23 overexpression reversed the effects of circPVTI silence on HCC cell progression. SNU-387 and Huh7 cells were transfected with si-NC, si-circPVTI, sicircPVTI + pcDNA-Control or si-circPVTI + pcDNA-TRIM23. (A and B) The mRNA and protein levels of TRIM23 in SNU-387 and Huh7 cells after transfection were explored by qRT-PCR assay and Western blot assay, respectively. (C and D) Cell proliferation in SNU-387 and Huh7 cells after transfection was tested by MTT assay. (E) The apoptosis rate of SNU-387 and Huh7 cells was examined by flow cytometry. $* P<0.05$.

The results showed that the decreased mRNA and protein expression of TRIM23 in SNU-387 and Huh7 cells induced by circPVT1 knockdown was abolished by miR-377 inhibitor (Figure 6A and B). These data revealed that circPVT1 modulated TRIM23 expression by sponging miR-377 in HCC cells.

\section{CircPVTI Accelerated Glycolysis in HCC Cells by Reducing miR-377 and Enhancing TRIM23 Expression}

Many studies have attested that the accelerated glycolysis metabolism is a remarkable feature of energy metabolism in cancer cells and participates in tumorigenesis. ${ }^{17}$ The
A

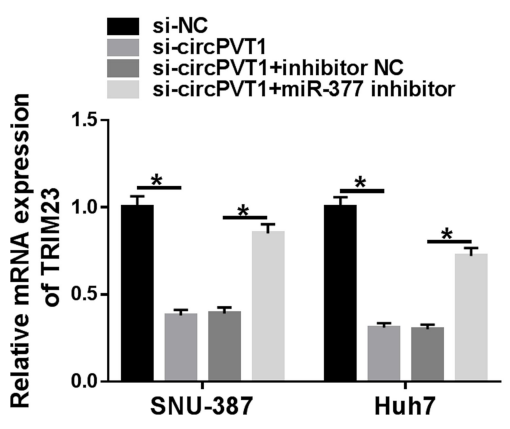

B

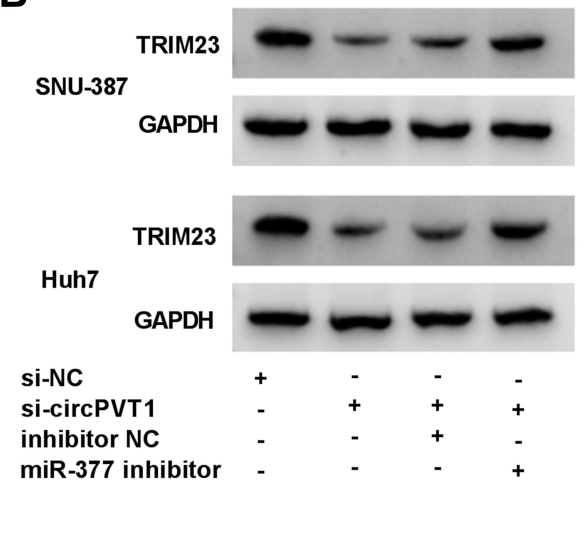

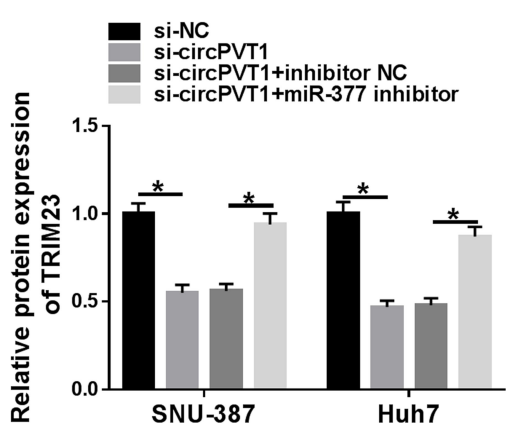

Figure 6 CircPVTI accelerated the expression of TRIM23 by targeting miR-377 in HCC cells. (A and B) The mRNA and protein levels of TRIM23 in SNU-387 and Huh7 cells transfected with si-circPVTI, si-circPVTI + miR-377 inhibitor or matched controls were determined by qRT-PCR assay and Western blot assay, respectively. $* P<0.05$. 
consumption of glucose in glycolysis of cancer cells can produce end-product lactate. ${ }^{17}$ Thus, we investigated whether circPVT1 affected glycolysis of HCC cells by detecting both glucose consumption and lactate production. The results indicated that deletion of circPVT1 drastically decreased glucose consumption and lactate production in SNU-387 and Huh7 cells (Figure 7A and B). As depicted in Figure 7C, D, G and H, the decreased glucose consumption and lactate production in SNU-387 and Huh7 cells induced by circPVT1 deletion were both restored by miR377 inhibition or TRIM23 overexpression. Moreover, TRIM23 overexpression also overturned the inhibitory effects of miR-377 mimic on glucose consumption and lactate production (Figure $7 \mathrm{E}$ and $\mathrm{F}$ ). In addition, the indicators of glycolysis (ECAR and OCR) were examined in this study. ECAR can reflect overall glycolytic flux, while OCR can reflect mitochondrial oxidative phosphorylation. ${ }^{17}$ The data suggested that downregulation of circPVT1 could significantly suppress ECAR and enhance OCR in both SNU-387 and Huh7 cells, while these effects were partly abrogated by miR-377 inhibitor (Figure 8A-D). Likewise, overexpression of TRIM23 distinctly restored the declined ECAR and elevated OCR caused by miR-377 overexpression in SNU-387 and Huh7 cells (Figure 8E-H). These data implied that circPVT1/miR-377/TRIM23 axis accelerated glycolysis in HCC cells.

\section{CircPVTI Knockdown Suppressed Tumor Growth in vivo}

To verify the effect of circPVT1 on tumor growth in vivo, SNU-387 cells stably transfected with sh-circPVT1 or sh$\mathrm{NC}$ were injected into the nude mice. The tumor volume and weight were markedly restrained in sh-circPVT1 group relative to the control group (Figure 9A and B). Besides, the levels of circPVT1, miR-377 and TRIM23 in the tumors were examined. The results showed that circPVT1 and TRIM23 levels were reduced, whereas the level of miR-377 was raised in tumor tissues collected from the mice of sh-circPVT1 group (Figure 9C-F). These in vivo data confirmed that circPVT1 promoted tumor growth in HCC through the miR-377/TRIM23 axis.

\section{Discussion}

In the recent years, a variety of circRNAs have been announced to serve as important regulators in $\mathrm{HCC}$ by regulating various biological processes. ${ }^{18,19}$ In this study, we mainly ascertained the expression trend and effect of circPVT1 on proliferation, apoptosis and glycolysis in HCC. Moreover, the circPVT1/miR-377/TRIM23 regulatory axis was constructed to expound the action mechanism of circPVT1 in $\mathrm{HCC}$, in an attempt to better understand the potential pathogenesis of $\mathrm{HCC}$ and present
A
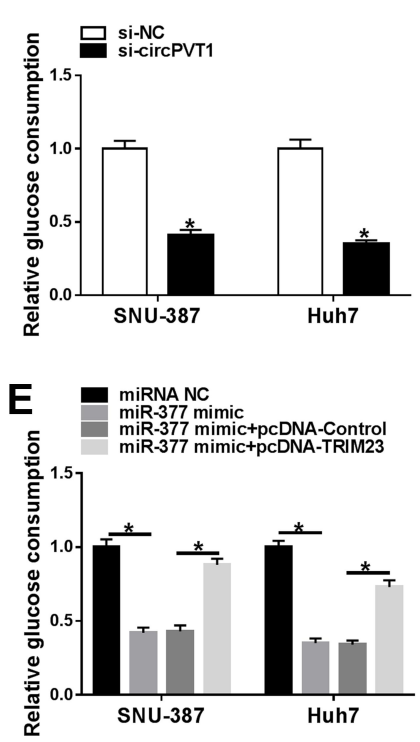

B
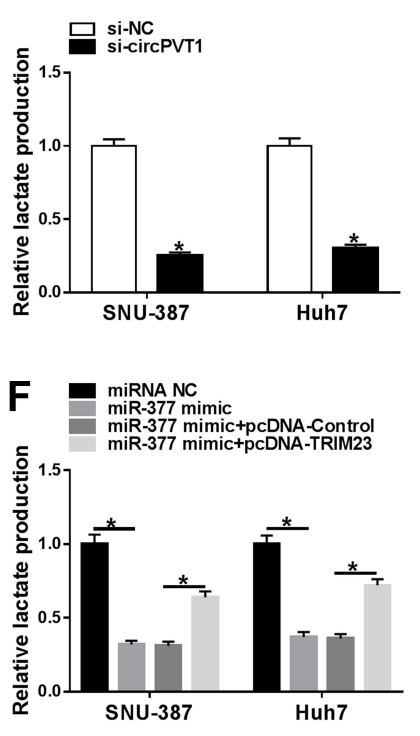

C
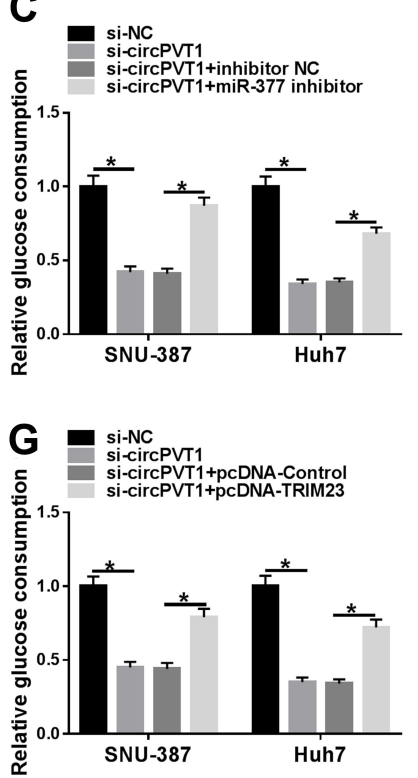
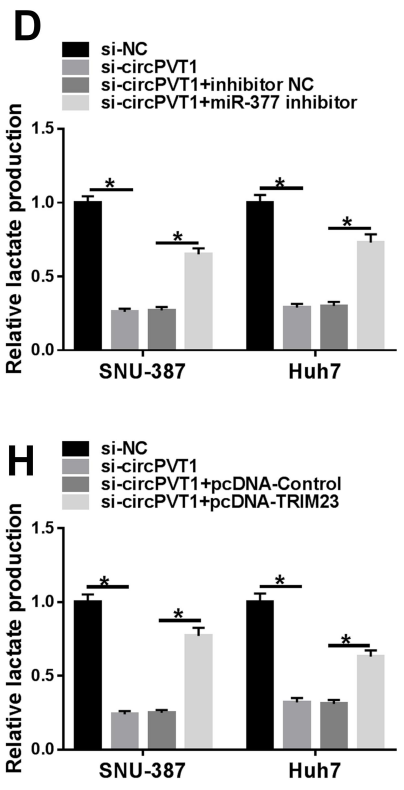

Figure 7 CircPVTI expedited glucose consumption and lactate production in HCC cells via miR-377/TRIM23 axis. (A and B) The glucose consumption and lactate production levels in SNU-387 and Huh7 cells after circPVTI knockdown were evaluated by specific kits. (C and D) The glucose consumption and lactate production levels in SNU-387 and Huh7 cells transfected with si-NC, si-circPVTI, si-circPVTI + miR-377 inhibitor, or si-circPVTI + miR-377 inhibitor were measured. (E-H) The glucose consumption and lactate production levels in SNU-387 and Huh7 cells transfected with miRNA NC, miR-377 mimic, miR-377 mimic + pcDNA-Control, or miR-377 mimic + pcDNA-TRIM23 were detected. $* P<0.05$. 

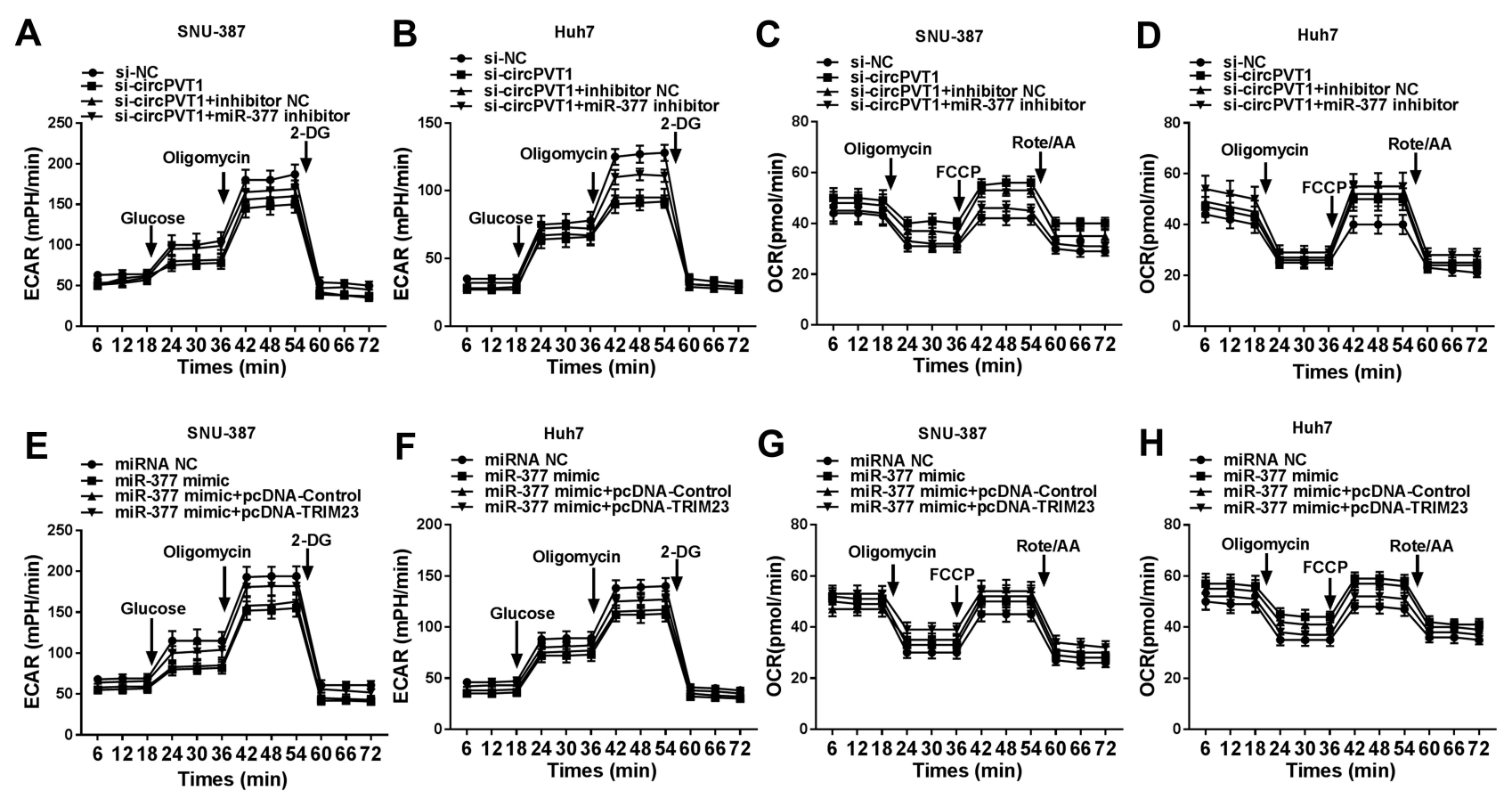

Figure 8 CircPVTI increased ECAR level and suppress OCR level in HCC cells by regulating miR-377 and TRIM23 expression. (A-D) The quantification of OCR and ECAR in SNU-387 and Huh7 cells transfected with si-NC, si-circPVTI, si-circPVTI + miR-377 inhibitor, or si-circPVTI + miR-377 inhibitor was measured by Seahorse Extracellular Flux Analyzer XF96. (E-H) The quantification of OCR and ECAR in SNU-387 and Huh7 cells transfected with miRNA NC, miR-377 mimic, miR-377 mimic + pcDNAControl, or miR-377 mimic + pcDNA-TRIM23 were determined.

the cancer-related biomarkers for diagnosis and treatment for HCC.

CricRNAs are a type of non-coding RNAs and 3' and $5^{\prime}$ ends of circRNAs are covalently connected to form a stable closed-loop structure. Accumulating evidence indicated that the expression alterations of circRNAs have an extensive influence in biological characteristics of HCC. ${ }^{18}$ For instance, circFBLIM1 might exert biological regulatory functions in $\mathrm{HCC}^{20} \mathrm{Hsa}$ _circ_0103809 accelerated proliferation and migration and impeded apoptosis in HCC by acting as a sponge of miR-490-5p. ${ }^{21}$ CircPVT1 was deemed to be an oncogenic noncoding RNA with emerging clinical importance. ${ }^{22}$ CircPVT1 was reported to be upregulated and could promote HCC cell proliferation by targeting miR-3666 and regulating Sirtuin 7 expression. ${ }^{23}$ Consistent with previous reports, we found that circPVT1 level was elevated in HCC tissues and cells, and circPVT1 silence hampered HCC progression in vitro and in vivo. All these results suggested that circPVT1 played an oncogenic role in HCC.

Meanwhile, it has been documented that the major mechanism of circRNAs was as competitive endogenous RNA (ceRNA) to sponge miRNA and then act on mRNA in multiple cancers. ${ }^{24-27}$ Starbase software was used to predict the binding sites between circPVT1 and miRNAs.
The result showed that circPVT1 directly targeted miR377. Moreover, we found that miR-377 was downregulated in HCC tissues and the influences of circPVT1 silence on HCC cells were all weakened by the inhibition of miR-377. All these data revealed that circPVT1 participated in the progression of HCC by sponging miR-377.

TRIM23, an ubiquitin ligase, was upregulated in many cancers, including gastric $\operatorname{cancer}^{28}$ and lung adenocarcinoma. ${ }^{29}$ However, studies about the specific expression and function of TRIM23 in HCC were very rare. It has been reported that TRIM 23 could facilitate the migration and invasion of HCC cells. ${ }^{30}$ Additionally, the circRNA-miRNA-mRNA network has been exhibited to be involved in HCC development. ${ }^{31}$ Similarly, miR-377 could hinder HCC progression by functioning as a target of circRNA and regulating the mRNA expression of downstream gene. For example, miR-377-3p regulated HCC proliferation, cycle progression, and migration through affecting FGFRlexpression. ${ }^{32}$ CircVAPA could contribute to HCC cell proliferation through sponging miR-377-3p to regulate PSAPexpression. ${ }^{5}$ In this research, it was revealed that miR-377 targeted TRIM23 and overexpression of TRIM23 abrogated miR-377 mimic-induced effects on HCC cells, implying that miR377 could decelerate HCC development via targeting 
A

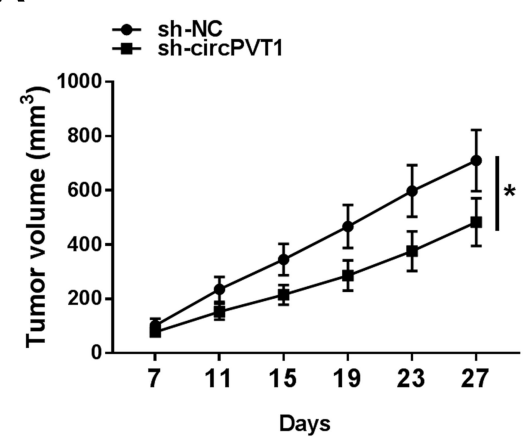

D

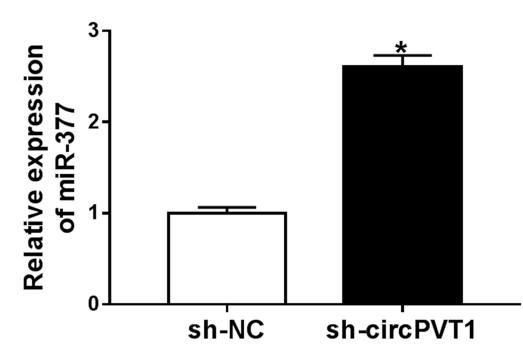

B
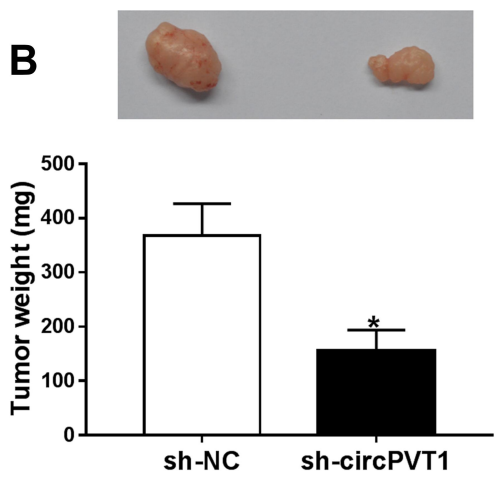

$\mathbf{E}$

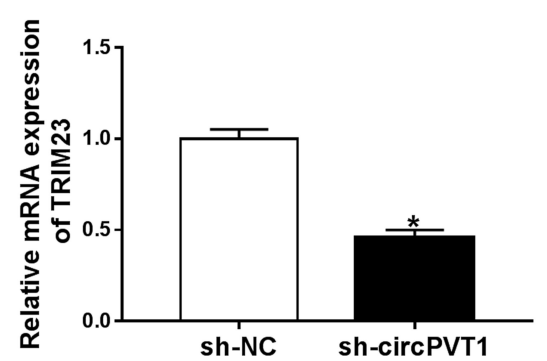

C
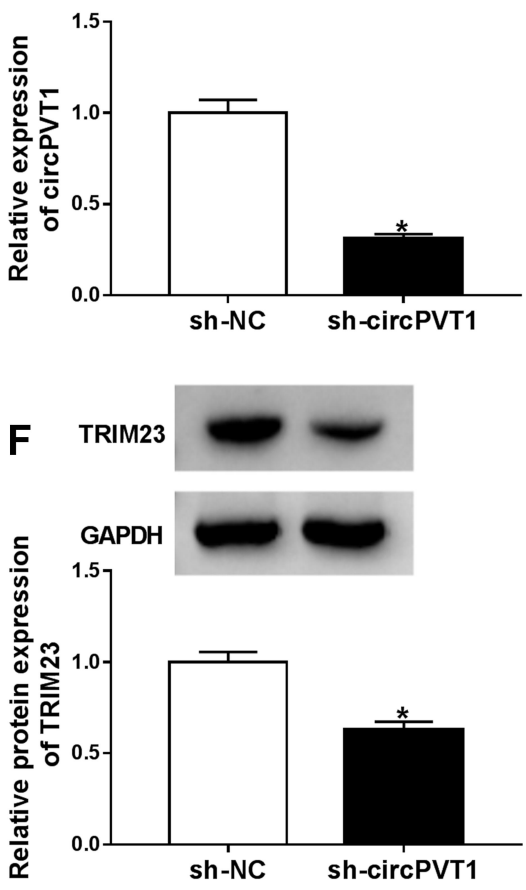

Figure 9 CircPVTI knockdown hindered tumor growth in vivo. Sh-circPVTI or sh-NC transfected SNU-387 cells were injected into the nude mice. (A) Tumor volume was monitored every 4 days. (B) Tumor weight was examined after cells were injected for 27 days. (C-E) The levels of circPVTI, miR-377 and TRIM23 mRNA in the xenograft tumors were determined by qRT-PCR. (F) The protein level of TRIM23 in the xenograft tumors was detected by Western blot assay. $* P<0.05$.

TRIM23. Moreover, TRIM23 overexpression also overturned the effects of circPVT1 silence on HCC cell progression. Thus, it was concluded that circPVT1 modulated the progression of HCC through the miR377/TRIM23 axis. Furthermore, circPVT1/miR-377/ TRIM23 can act as novel biomarkers and clinical targets for HCC.

\section{Conclusion}

In summary, circPVT1 expression was enhanced in HCC tissues and cells. Gain- or loss-function experiments concluded that the carcinogenesis of circPVT1 was accomplished by regulating proliferation, apoptosis and glycolysis via the TRIM23/miR-377 axis. These findings enriched our knowledge of HCC pathogenesis and contributed to the diagnosis and treatment of HCC.

\section{Highlights}

1. CircPVT1 knockdown suppresses HCC proliferation, glycolysis and accelerates apoptosis in HCC cells.

2. CircPVT1 silence decreases TRIM23 expression via sponging miR-377.
3. CircPVT1 deletion inhibited the progression of HCC through the miR-377/TRIM23 axis.

4. CircPVT1 knockdown impedes tumor growth in vivo.

\section{Funding}

This work was supported by High-level Talents Training Project on Health and Family Planning in Yunnan Province (Yunwei Science and Education Commission [2017] No. 14), Basic Research Joint Project for Local Universities in Yunnan Province (Part) (2018FH001-076, 2018FH001-080).

\section{Disclosure}

The authors declare that they have no financial or nonfinancial conflicts of interest.

\section{References}

1. Chen S, Cao Q, Wen W, et al. Targeted therapy for hepatocellular carcinoma: challenges and opportunities. Cancer Lett. 2019;460 (1):1-9. doi:10.1016/j.canlet.2019.114428

2. Chien Y, Tsai PH, Lai YH, et al. CircularRNA as novel biomarkers in liver diseases. J Chin Med Assoc. 2019:1. doi:10.1097/ JCMA.0000000000000230 
3. Shang Q, Yang Z, Jia R, et al. The novel roles of circRNAs in human cancer. Mol Cancer. 2019;18(1):6. doi:10.1186/s12943-018-0934-6

4. Luo Z, Mao X, Cui W. Circular RNA expression and circPTPRM promotes proliferation and migration in hepatocellular carcinoma. Med Oncol. 2019;36(10):86. doi:10.1007/s12032-019-1311-z

5. Liu C, Zhong X, Li J, et al. Circular RNA circVAPA promotes cell proliferation in hepatocellular carcinoma. Hum Gene Ther Clin Dev. 2019;30(4):152-159. doi:10.1089/humc.2019.079

6. He T, Li X, Xie D, et al. Overexpressed circPVT1 in oral squamous cell carcinoma promotes proliferation by serving as a miRNA sponge.Mol Med Rep. 2019;20(4):3509-3518.

7. Zhong R, Chen Z, Mo T, et al. Potential role of circPVT1 as a proliferative factor and treatment target in esophageal carcinoma. Cancer Cell Int. 2019;19(267). doi:10.1186/s12935-019-0985-9.

8. Wang Z, Su M, Xiang B, et al. Circular RNA PVT1 promotes metastasis via miR-145 sponging in CRC. Biochem Biophys Res Commun. 2019;512(4):716-722. doi:10.1016/j.bbrc.2019.03.121

9. Zhu Y, Liu Y, Xiao B, et al. The circular RNA PVT1/miR-203/ HOXD3 pathway promotes the progression of human hepatocellular carcinoma. Biol Open. 2019;8(9):bio043687. doi:10.1242/bio.043687

10. Bartel DP. MicroRNAs: genomics, biogenesis, mechanism, and function. Cell. 2004;116(2):281-297. doi:10.1016/S0092-8674(04) 00045-5

11. Wen X, Wu JQ, Peng W, et al. MicroRNA-377 predicts poor clinical outcome of gastric cancer and induces tumorigenesis by targeting multiple tumor-suppressor genes. Oncol Rep. 2015;34(1):203-210. doi:10.3892/or.2015.3981

12. Liu WY, Yang Z, Sun Q, et al. miR-377-3p drives malignancy characteristics via upregulating GSK-3beta expression and activating NF-kappaB pathway in hCRC cells. J Cell Biochem. 2018;119 (2):2124-2134. doi:10.1002/jcb.26374

13. Xia P, Gu R, Zhang W, et al. MicroRNA-377 exerts a potent suppressive role in osteosarcoma through the involvement of the histone acetyltransferase 1-mediated Wnt axis. J Cell Physiol. 2019;234 (12):22787-22798. doi:10.1002/jcp.28843

14. Ge H, Zou D, Wang Y, et al. MicroRNA-377 downregulates Bcl-xL and increases apoptosis in hepatocellular carcinoma cells. Oncol Res. 2017;25(1):29-34. doi:10.3727/096504016X14719078133168

15. Chen G, Lu L, Liu C, et al. MicroRNA-377 suppresses cell proliferation and invasion by inhibiting TIAM1 expression in hepatocellular carcinoma. PLoS One. 2015;10(3):e0117714. doi:10.1371/journal. pone. 0117714

16. Hatakeyama S. TRIM proteins and cancer. Nat Rev Cancer. 2011;11 (11):792-804. doi:10.1038/nrc3139

17. Ganapathy-Kanniappan S, Geschwind JF. Tumor glycolysis as a target for cancer therapy: progress and prospects. Mol Cancer. 2013;12(1):152. doi:10.1186/1476-4598-12-152

18. Wang M, Yu F, Li P. Circular RNAs: characteristics, function and clinical significance in hepatocellular carcinoma. Cancers (Basel). 2018;10(8):258. doi:10.3390/cancers 10080258
19. Qiu L, Xu H, Ji M, et al. Circular RNAs in hepatocellular carcinoma: biomarkers, functions and mechanisms. Life Sci. 2019;231:116660. doi:10.1016/j.lfs.2019.116660

20. Bai N, Peng E, Qiu X, et al. circFBLIM1 act as a ceRNA to promote hepatocellular cancer progression by sponging miR-346. J Exp Clin Cancer Res. 2018;37(1):172. doi:10.1186/s13046-018-0838-8

21. Cai H, Hu B, Ji L, et al. Hsa_circ_0103809 promotes cell proliferation and inhibits apoptosis in hepatocellular carcinoma by targeting miR-490-5p/SOX2 signaling pathway. Am J Transl Res. 2018;10 (6):1690-1702.

22. Adhikary J, Chakraborty S, Dalal S, et al. Circular PVT1: an oncogenic non-coding RNA with emerging clinical importance. $J$ Clin Pathol. 2019;72(8):513-519. doi:10.1136/jclinpath-2019-205891

23. Li Y, Shi H, Yuan J, Qiao L, Dong L, Wang Y. Downregulation of circular RNA circPVT1 restricts cell growth of hepatocellular carcinoma through downregulation of Sirtuin 7 via microRNA-3666. Clin Exp Pharmacol Physiol. 2020;47(7):1291-1300. doi:10.1111/14401681.13273

24. Chen Y, Sun F, Zhang Y, et al. Comprehensive molecular characterization of circRNA-associated ceRNA network in constrictive pericarditis. Ann Transl Med. 2020;8(8):549. doi:10.21037/atm-202912

25. Qi X, Zhang DH, Wu N, Xiao JH, Wang X, Ma W. ceRNA in cancer: possible functions and clinical implications. J Med Genet. 2015;52 (10):710-718. doi:10.1136/jmedgenet-2015-103334

26. Liu L, Wu SQ, Zhu X, et al. Analysis of ceRNA network identifies prognostic circRNA biomarkers in bladder cancer. Neoplasma. 2019;66(5):736-745. doi:10.4149/neo_2019_190107N25

27. Gong J, Jiang $\mathrm{H}$, Shu $\mathrm{C}$, et al. Integrated analysis of circular RNA-associated ceRNA network in cervical cancer: observational study. Medicine (Baltimore). 2019;98(34):e16922. doi:10.1097/ MD.0000000000016922

28. Yao Y, Liu Z, Guo H, et al. Elevated TRIM23 expression predicts poor prognosis in Chinese gastric cancer. Pathol Res Pract. 2018;214 (12):2062-2068. doi:10.1016/j.prp.2018.10.010

29. Zhang Y, Du H, Li Y, et al. Elevated TRIM23 expression predicts cisplatin resistance in lung adenocarcinoma. Cancer Sci. 2019.

30. Bao C, Li Y, Huan L, et al. NF-kappaB signaling relieves negative regulation by miR-194 in hepatocellular carcinoma by suppressing the transcription factor HNF-1alpha.Sci Signal. 2015;8(387):ra75. doi:10.1126/scisignal.aaa8441

31. Xiong DD, Dang YW, Lin P, et al. A circRNA-miRNA-mRNA network identification for exploring underlying pathogenesis and therapy strategy of hepatocellular carcinoma. J Transl Med. 2018;16 (1):220. doi:10.1186/s12967-018-1593-5

32. Zhan W, Liao X, Chen Z, et al. Circular RNA hsa_circRNA_103809 promoted hepatocellular carcinoma development by regulating miR-377-3p/FGFR1/ERK axis.. $J$ Cell Physiol. 2020;235 (2):1733-1745. doi:10.1002/jcp.29092

\section{Publish your work in this journal}

Cancer Management and Research is an international, peer-reviewed open access journal focusing on cancer research and the optimal use of preventative and integrated treatment interventions to achieve improved outcomes, enhanced survival and quality of life for the cancer patient.
The manuscript management system is completely online and includes a very quick and fair peer-review system, which is all easy to use. Visit http://www.dovepress.com/testimonials.php to read real quotes from published authors. 